$\$=$

\title{
Birth outcomes of cases with different congenital heart defects - a population-based study
}

\author{
Attila Vereczkey ${ }^{1 *}$, Balázs Gerencsér ${ }^{2}$, Andrew E. Czeizel ${ }^{3}$, István Szabó ${ }^{4}$ \\ ${ }^{1}$ Versys Clinics, Human Reproduction Institute, Budapest, 1138, Madarász Viktor utca 47-49, Hungary \\ ${ }^{2}$ Alfréd Rényi Institute of Mathematics, Hungarian Academy of Science, Budapest, 1053, Reáltanoda utca 13-15, Hungary \\ ${ }^{3}$ Foundation for the Community Control of Hereditary Diseases, Budapest, 1026, Törökvész lejtő 32, Hungary \\ ${ }^{4}$ Department of Obstetrics and Gynecology, University of Pécs, Pécs, 7624, Édesanyák útja 17, Hungary \\ *Corresponding author E-mail: attila.vereczkey@versysclinics.com
}

\begin{abstract}
Background: In general, previous epidemiological studies evaluated congenital heart defects (CHDs) together. The aim of the present study was to identify possible etiological factors of different CHD-entities, because the underlying causes are unclear in the vast majority of patients.

Objectives: Different CHD-entities as homogeneously as possible with confirmed diagnoses were analyzed in the population-based large dataset of the Hungarian Case-Control Surveillance of Congenital Abnormalities.

Methods: 3,750 live-born singleton CHD-patients were analyzed according to birth outcomes, i.e. gestational age at delivery and birth weight, the rate of preterm birth, low birthweight and small for gestational age.

Results: The major findings of the study showed that cases with different CHD-entities had shorter gestational age at delivery and lower mean birth weight, and these variables associated with a higher rate of preterm birth and particularly with a much higher rate of low birthweight and small for gestational age. This study showed the importance of sex in the birth outcomes of some CHD-entities. The question is why several CHD-entities manifested more frequently in newborns with intrauterine growth restriction because fetal heart has a passive role before birth without pulmonary circulation.

Conclusions: The birth outcomes of cases indicate the effect of CHDs for fetal development. In addition maternal confounders have to consider. Finally, CHDs and intrauterine growth restriction as two developmental errors may have a common route, thus fetal growth and birthweight associated gene polymorphisms may have a role in the origin of CHDs.
\end{abstract}

Keywords: Congenital Heart Defects; Low Birthweight; Population-Based Study; Preterm Birth; Small for Gestational Age.

\section{Introduction}

The aim of our project is to reveal the possible environmental risk factors in the origin of CHDs in the population-based Hungarian Case-Control Surveillance of Congenital Abnormalities (HCCSCA) (Czeizel et al. 2001). However, CHDs cannot be regarded as a single homogeneous CA (Botto et al. 2007) therefore; CHD-entities/groups were separated according to the classification of CHDs used in the Baltimore-Washington Infant Study (Ferencz et al. 1993, Ferencz et al. 1997). In addition syndromic/multiple CAs including CHD were excluded from the study material and only the serious cases with confirmed diagnosis due to lethal outcome or catheter diagnosis or surgical correction were included in the study and CHD-entities were evaluated as homogeneous CHD-entities as possible. This approach needs the previous analysis of birth outcomes of patients with CHD, and these data are presented here based on the HCCSCA (Czeizel et al. 2001).

\section{Methods}

\subsection{The Hungarian Congenital Abnormality Registry (HCAR)}

The HCAR was established as the first national-based registry of CAs in the world, 1962 (Czeizel 1997, Czeizel et al. 2014). Reporting of patients as cases with CA to the HCAR is mandatory for physicians from the birth until the end of first postnatal year. Most cases are reported by obstetricians (in Hungary practically all deliveries occur in inpatient obstetric clinics and birth attendants are obstetricians) and pediatricians (who are working in the neonatal units of inpatient obstetric clinics and various, general and specialized, e.g. cardiologic inpatient and outpatient pediatric clinics). Autopsy is mandatory for all infant deaths and common $(80 \%)$ in stillborn fetuses during the study period, and pathologists send a copy of the autopsy report to the HCAR if defects were identified. Since 1984, prenatal diagnostic centers were also asked to report malformed fetuses diagnosed prenatally with or without elective termination of pregnancy to the HCAR.

In the HCAR cases with CAs are differentiated into isolated (including single, sequence and complex groups) and multiple or syndromic (including CA-syndromes, CA-associations and 
unclassified multiple CAs) categories (Czeizel et al. 1993). The multimalformed cases had 2 or more CAs in different organs with or without minor anomalies. Thus the unit of recording was the person and not the CA in the HCAR. The recorded total (birth + fetal) prevalence rate of cases with CA was 35 per 1000 informative offspring (live-born infants, stillborn fetuses and electively terminated malformed fetuses) between 1980 and 1996 (Czeizel 1997) and about 90\% of major CAs were recorded in the HCAR (Czeizel et al. 1993).

\subsection{The Hungarian Case-Control Surveillance of Con- genital Abnormalities (HCCSCA)}

Cases with CA including CHD in the HCCSCA were selected from the HCAR. Only those CA cases were selected from the HCAR for the HCCSCA that were reported to the HCAR during the first three months after birth or elective termination of pregnancy. In addition cases with CA-syndromes caused by gene mutations or chromosomal aberrations with preconception origin were excluded.

Population controls were defined as newborn infants without CAs and were selected from the National Birth Registry of the Central Statistical Office for the HCCSCA based on case lists for each quarter of the years from the HCAR. In general, two controls were matched to every case according to sex, birth week, and district of parents' residence. If controls were twins, only one of them was randomly selected as controls for the HCCSCA.

A letter with informed consent was mailed continuously to the address of the mothers of cases and controls immediately after their selection for the HCCSCA and requested that they send us the prenatal maternity logbook, discharge summary of their delivery and every medical record concerning their pregnancy and their child's CA. These documents were sent back within four weeks.

The mean + S.D. time elapsed between the end of pregnancy and return of the "information package" (including logbook, discharge summary, signed informed consent, etc.) in our prepaid envelope was $3.5+2.1$ and $5.2+2.9$ months in cases and population controls, respectively.

In addition regional district nurses were asked to visit all nonrespondent case mothers and to evaluate the available medical documents. Unfortunately, district nurses could visit only 200 non-respondent (Czeizel et al. 2003) and 600 respondent (Czeizel $\&$ Vargha 2004) control mothers in two validation studies because the ethics committee considered this follow-up to be disturbing for the parents of all healthy children.

The necessary information was available for $96.3 \%$ of cases (84.4\% from replies and $11.9 \%$ from visits) and $83.0 \%$ of population controls ( $81.3 \%$ from replies and $1.7 \%$ from visits) The signed informed consent was sent back by $98 \%$ of mothers. The name and address were deleted in $2 \%$ of subjects without signed informed consent. The flow of cases and population controls in the HCCSCA was reported previously (Ács et al. 2005). The data of sex and birth outcomes in cases were based on the Notification Form of Cases with CA in the HCAR confirmed by the discharge summary of delivery in the HCCSCA. The sex and birth outcomes of population controls were evaluated by the help of the discharge summary of delivery in the HCCSCA. The gestational age at delivery was calculated in a week from the first day of the last menstrual period while the definition of preterm birth (PTB) was as the time of birth of live-born babies in less than 37 completed gestation weeks. The rate of low birthweight (LBW) newborns (less than 2500 gram) was calculated based on birth weight, while the definition of small for gestational age (SGA) was birth weight less than $2500 \mathrm{~g}$ in newborn infants born on 37 th gestational week or later.

The method of data collection was changed in 1997 (since all case and control mothers are visited and questioned at home by regional nurses, but these data have not been validated at the time of this analysis), and it explains that here only the 17 years' dataset of the HCCSCA, 1980-1996 are evaluated.

\subsection{Study design of cases with CHD}

The major evaluation problem of CHDs was that in general cases with $\mathrm{CA}$ were reported immediately after birth to the HCAR and about $50 \%$ of cases with CHD were reported as unspecified CHD, because the exact diagnosis of CHDs needed further time consuming examinations. The collection of medical data of cases with $\mathrm{CA}$ in the HCCSCA was $3.5+2.1$ months later thus, we could get specified CHD diagnoses in further $20 \%$ of cases. The remaining $30 \%$ of CHD cases with unspecified diagnosis were expected to have been cared for or had surgical intervention in the pediatric cardiologic institutions in Hungary. Thus the staff of the HCCSCA visited the cardiologic in- and outpatient clinics in 2008 and reviewed the medical records of patients. In cases with unspecified CHD, wherever possible, the diagnoses were modified to specific CHD. In addition, the previous diagnosis of CHD was checked and sometimes modified. If previously reported cases were not identified in the records of pediatric cardiologic institutions, we corresponded with mothers to clarify the fate and/or diagnosis of these cases in 2009 and 2010. However, if these mothers were not found or cases had further unspecified $\mathrm{CHD}$, or mothers refused to collaborate, the cases were excluded from the study.

There were three selection steps in the evaluation of cases with CHD:

i) Cases with syndromic CHD due to major mutant genes (e.g. Holt-Oram) or chromosomal aberrations (e.g. Down) were excluded from the HCCSCA, and unclassified multiple CAs including CHD were also excluded from the study.

ii) Cases with CHD were classified according to the system of The Baltimore-Washington Infant Study (Ferencz et al. 1993, Ferencz et al. 1997), including homogeneous structural entities/groups of CHD with a sufficient number of cases.

iii) Some types of CHD have a wide spectrum of manifestations, including spontaneous closure of ventricular or atrial septal defects, ductus arteriosus, etc. Thus finally only cases with lethal outcomes verified by autopsy record or with documented catheter diagnoses or surgical corrections were included in the study.

Our cases, therefore, represent homogeneous groups of severe manifestations of different entities of CHD.

\subsection{Statistical analysis}

The software GNU R 2.14, RStudio 0.97 version was used for the statistical analysis of data. At the evaluation of quantitative birth outcomes such as gestational age at delivery and birth weight of newborn infants, Student t-test was used. At the evaluation of categorical variables: PTB, LBW and SGA were analyzed by odds ratios (OR) with $95 \%$ confidence intervals $(\mathrm{CI})$ in a multivariable unconditional regression model at the comparison of cases and population controls. At the calculation of adjusted OR, maternal age and birth order were considered.

\section{Results}

The number of cases with different CHD-entities/groups and their abbreviations are shown in Table 1 . Of 22 CHDs, 17 were CHDentities, however. 4-4 entities were combined as CTD, RSOD and LSOD groups, respectively, and other rare CHDs were evaluated together in the group of OCHDs while other unspecified complex CHDs (e.g. VSD + ASD) in the group of CCHD. The birth outcomes of the latter two CHD-groups including heterogeneous CHDs will not be evaluated here in detail.

Finally, 3,849 cases with confirmed diagnosis were evaluated, but 15 occurred in stillborn fetuses. The sex (percentage proportion of boys) and twin rate (\%) of different CHD-entities/groups were 
analyzed in 3,834 live-born cases compared to the Hungarian newborns as reference $(51.3 \%)$ in Table 1 . The proportion of boys was $50.7 \%$, while the rate of twin deliveries was $2.2 \%$ in the Hungarian newborns. The sex of cases with total CHD was near to the population reference figure, but their rate of twins was 2 fold higher.

The evaluation of birth outcomes of cases with CHD was based on singletons because 84 twin cases had drastically shorter gestational age and smaller birth weight. Thus finally 3,750 singleton live-born cases with different CHD-entities and groups were evaluated. Our population-based data set included 37,741 singleton live-born population controls without $\mathrm{CA}, 1.8 \%$ of all live-births in Hungary and their birth outcomes corresponded well to the data of the Hungarian newborn population. Beyond sex of newborns, maternal age and birth order also have effect for birth outcomes. At the calculation of adjusted $\mathrm{p}$ value and OR, sex of cases and population controls, maternal age and birth order were considered (Table 2).

Table 1: Number of Live-Births, Boys and Twins in Different Study Groups

\begin{tabular}{|c|c|c|c|c|c|c|}
\hline \multirow{2}{*}{ Study groups } & \multirow{2}{*}{ Abbreviation } & \multirow{2}{*}{ Total N } & \multicolumn{2}{|l|}{ Boys } & \multicolumn{2}{|l|}{ Twins } \\
\hline & & & No. & $\%$ & No. & $\%$ \\
\hline Hungarian newborns & & $2,134,150$ & $1,094,017$ & 51.3 & 1,088 & 1.1 \\
\hline Ventricular septal defect* & VSD & 1,659 & 801 & 48.3 & 28 & 1.7 \\
\hline Single ventricle & SV & 76 & 40 & 52.6 & 4 & 5.3 \\
\hline Atrial septal defect secundum & ASD-II & 471 & 221 & 46.9 & 17 & 3.6 \\
\hline Common atrioventricular canal & CAVC & 79 & 32 & 40.5 & 2 & 2.5 \\
\hline Patent ductus arteriosus & PDA & 181 & 77 & 42.5 & 6 & 3.3 \\
\hline Common truncus & $\mathrm{CT}$ & 44 & 31 & 70.5 & 1 & 2.3 \\
\hline Transposition of great arteries & TGA & 307 & 169 & 55.0 & 5 & 1.6 \\
\hline Tetralogy of Fallot & $\mathrm{TF}$ & 222 & 127 & 57.2 & 6 & 2.7 \\
\hline Double outlet of right ventricle & DORV & 24 & 12 & 50.0 & 0 & 0.0 \\
\hline Conotruncal defects & CTD & 597 & 339 & 56.8 & 12 & 2.0 \\
\hline Atresia/stenosis of pulmonary valve & $\mathrm{A} / \mathrm{SPV}$ & 72 & 37 & 51.4 & 0 & 0.0 \\
\hline Atresia/stenosis of tricuspid valve & $\mathrm{A} / \mathrm{STV}$ & 13 & 5 & 38.5 & 0 & 0.0 \\
\hline Ebstein's anomaly & EA & 7 & 6 & 85.7 & 0 & 0.0 \\
\hline CAs of pulmonary artery & CAPA & 108 & 60 & 55.6 & 1 & 0.9 \\
\hline Right sided obstructive defects & RSOD & 200 & 108 & 54.0 & 1 & 0.5 \\
\hline Valvular aortic stenosis & VAS & 56 & 37 & 66.1 & 0 & 0.0 \\
\hline Hypoplastic left heart & $\mathrm{HLH}$ & 76 & 49 & 64.6 & 3 & 3.9 \\
\hline Coarctation of aorta & $\mathrm{COA}$ & 113 & 71 & 62.8 & 1 & 0.9 \\
\hline Other CAs of aorta & OCAA & 57 & 38 & 66.7 & 3 & 5.3 \\
\hline Left sided obstructive defects & LSOD & 302 & 195 & 64.6 & 7 & 2.3 \\
\hline Other isolated CHD & OCHD & 186 & 86 & 46.2 & 5 & 2.7 \\
\hline Other complex CHD & $\mathrm{CCHD}$ & 83 & 44 & 53.0 & 2 & 0.2 \\
\hline Total & CHD & 3,834 & 1943 & 50.7 & 84 & 2.2 \\
\hline
\end{tabular}

*perimembranous and muscular ventricular septal defects

Table 2: Mean Maternal Age and Birth Order in Groups of Singleton Live-Born Cases with Different CHD Groups/Entities and Population Controls (PC) in the HCCSCA.

\begin{tabular}{|c|c|c|c|c|c|}
\hline \multirow{2}{*}{ Study groups } & \multirow{2}{*}{$\begin{array}{c}\text { Total } \\
\mathrm{N}\end{array}$} & \multicolumn{2}{|c|}{ Maternal age } & \multicolumn{2}{|c|}{ Birth order } \\
\hline & & Mean & S.D. & Mean & S.D. \\
\hline $\mathrm{PC}$ & 37,741 & 25.4 & 4.9 & 1.7 & 0.9 \\
\hline VSD & 1,631 & 25.6 & 5.2 & 1.9 & 1.2 \\
\hline ASD-II & 454 & 26.0 & 5.8 & 2.1 & 1.3 \\
\hline CAVC & 77 & 26.4 & 5.4 & 2.1 & 1.3 \\
\hline PDA & 175 & 25.7 & 5.2 & 2.0 & 1.4 \\
\hline $\mathrm{CT}$ & 43 & 25.1 & 4.2 & 2.0 & 1.2 \\
\hline TGA & 302 & 26.0 & 5.0 & 1.9 & 1.2 \\
\hline $\mathrm{TF}$ & 216 & 25.2 & 5.3 & 1.8 & 1.0 \\
\hline DORV & 24 & 26.6 & 4.3 & 2.0 & 0.8 \\
\hline CTD & 585 & 25.7 & 5.1 & 1.9 & 1.1 \\
\hline $\mathrm{A} / \mathrm{SPV}$ & 72 & 25.8 & 5.2 & 1.9 & 1.2 \\
\hline $\mathrm{A} / \mathrm{STV}$ & 13 & 25.0 & 4.1 & 1.7 & 1.0 \\
\hline EA & 7 & 24.3 & 7.6 & 1.6 & 1.1 \\
\hline CAPA & 107 & 26.3 & 5.9 & 2.1 & 1.3 \\
\hline RSOD & 199 & 26.0 & 5.6 & 2.0 & 1.2 \\
\hline HLH & 73 & 25.9 & 5.4 & 1.8 & 1.0 \\
\hline $\mathrm{COA}$ & 112 & 25.9 & 5.6 & 2.0 & 1.0 \\
\hline OCAA & 54 & 26.4 & 6.1 & 1.9 & 0.9 \\
\hline LSOD & 295 & 25.8 & 5.4 & 1.9 & 1.0 \\
\hline OCHD & 181 & 26.1 & 5.7 & 2.0 & 1.3 \\
\hline $\mathrm{CCHD}$ & 81 & 25.9 & 5.3 & 1.8 & 1.0 \\
\hline
\end{tabular}

Mean gestational age and birth weight of cases with different CHD entities/groups were compared to population controls, and differences were expressed in p values (Table 3). Cases with EA are not evaluated due to their limited number, thus the birth outcomes of 16 CHD-entities are analyzed.

The first impression is that the severity of CHD-entities did not correlate with the shorter mean gestational age: there is no difference in mean gestational age between severe TGA, DORV, $\mathrm{SV}, \mathrm{TF}$ and population controls, while the shortest gestational age was observed in cases with A/STV, ASD-II, OCAA, and PDA.
All 16 CHD-entities had lower mean birth weight than population controls; the difference did not reach the level of significance only in cases with DORV. The shortest gestational age associated with a lower mean birth weight in cases with A/STV, PDA, ASD-II, and OCAA. The largest mean birth weight was recorded in the groups of TGA and DORV. Another impression is that the wellknown relation between gestational age and birth weight is not obvious in some CHD-entities. For example, the combined mean gestational age was $39.4 \mathrm{wk}$ in the group of cases with CTD, similar to the figure of population controls, nevertheless their 
mean birth weight was lower nearly $200 \mathrm{~g}$ due to mainly CT and TF.

Mean gestational age and birth weight were evaluated in male and female singletons separately as well (Table 3 ). In the population control group, the mean gestational age was $0.1 \mathrm{wk}$ shorter while the mean birth weight was $137 \mathrm{~g}$ smaller in males than in females. In general mean gestational age was shorter and mean birth weight was lower in both sexes of cases than in population controls. However, there were four exceptions (i) there was a longer gestational age in both male and female cases with TGA, (ii) mean gestational age was longer in female cases with DORV than in population controls, (iii) the mean birth weight was larger in male cases with DORV and (iv) mean birth weight was similar in males with A/SPV to the mean birth weight in the male population controls. The mean birth weight of female cases was smaller than in population controls in all CHD-entities/groups.

Table 4 summarized the figures of categorical birth outcomes, i.e. rate of PTB, LBW and SGA in cases with different CHDentities/groups compared with the figures of population controls, and the differences are expressed in OR with 95\% CI. Of 16 CHD-entities, 6 (PDA, ASD-II, CAVC. HLH, CAPA and VSD) had higher rate of PTB. Of 16 CHD-entities, 14 showed higher rate of LBW but the two exceptional (DORV and VAS) had also higher rate of LBW, but the difference did not reach the level of significance though was near to it. The rate of SGA was also higher in most CHD-entities. Only four LSOD-entities were exceptional.

In the next step, categorical birth outcomes of cases were differentiated according to sex (Table 5 and 6). ASD-II and PDA showed a higher rate of PTB in both sexes, while it occurred only in females with CAVC. The higher rate of LBW was observed in most CHD-entities of both sexes, particularly in male cases. Only VAS was exceptional in both sexes, in addition TGA, DORV and A/SPV in males, SV. A/STV, COA and OCAA in females did not show a significantly higher rate of LBW. The rate of SGA was also higher in most CHD-entities, except in male cases with DORV, A/SPV and three entities of LSOD: VAS, HLH, COA and female cases with SV, CAVC, A/STV and four entities of LSOD.

The rate of PTB was higher in male cases while LWB and SGA were higher in females. In cases with VSD, ASD-II, RSOG and LSOD groups, the rate of PTB, LBW and SGA was higher in females than in males. The rate of LBW and SGA was higher in female cases with PDA and CTD group, but the rate of PTB was higher in males.

Table 3: Number of Singletons, in Addition Male and Female Singletons and Their Mean Gestational Age at Delivery and Mean Birth Weight in Different Study Groups and Population Controls (PC) As Reference (R)

\begin{tabular}{|c|c|c|c|c|c|c|c|c|c|c|c|c|c|c|c|c|c|c|c|c|}
\hline \multirow[t]{2}{*}{ Study Groups } & \multirow[t]{2}{*}{ Total N } & \multicolumn{3}{|c|}{ Gestational age } & \multicolumn{2}{|c|}{ Birth weight } & \multicolumn{4}{|c|}{ Male gestational age } & \multicolumn{3}{|c|}{ Male birth weight } & \multicolumn{4}{|c|}{ Female gestational age } & \multicolumn{3}{|c|}{ Female birth weight } \\
\hline & & Mean & S.D & $\mathrm{p}=$ & Mean S.D & $\mathrm{p}=$ & $\mathrm{N}$ & Mean & S.D. & $\mathrm{p}=$ & Mean & S.D. & $\mathrm{p}=$ & & Mean $S$ & S.D. & $p=$ & Mean & S.D. & $\mathrm{p}=$ \\
\hline $\mathrm{PC}$ & 37,741 & 39.4 & 2.0 & $\mathrm{R}$ & 3,282506 & $\mathrm{R}$ & 24,5 & 3639.4 & 2.0 & $\mathrm{R}$ & 3,330 & 508 & $\mathrm{R}$ & 13,20 & 539.32 & 2.1 & $\mathrm{R}$ & 3,193 & 489 & $\mathrm{R}$ \\
\hline VSD & 1,631 & 39.1 & 2.3 & 0.000 & 3,059590 & 0.000 & 784 & 39.2 & 2.3 & 0.001 & 3,141 & 608 & 0.000 & 847 & 39.12 & 2.3 & 0.187 & 2,983 & 564 & 0.000 \\
\hline SV & 72 & 39.3 & 2.4 & 0.918 & 3,090549 & 0.003 & 38 & 39.0 & 2.4 & 0.246 & 3,136 & 590 & 0.027 & 34 & 39.62 & 2.4 & 0.252 & 3,038 & 504 & 0.117 \\
\hline ASD-II & 454 & 38.2 & 3.1 & 0.000 & 2,870737 & 0.000 & 218 & 38.3 & 2.9 & 0.000 & 2,986 & 699 & 0.000 & 236 & 38.13 & 3.3 & 0.000 & 2,762 & 757 & 0.000 \\
\hline CAVC & 77 & 38.7 & 2.5 & 0.006 & $3,024 \quad 635$ & 0.000 & 31 & 39.1 & 2.2 & 0.530 & 3,176 & 666 & 0.102 & 46 & 38.42 & 2.6 & 0.006 & 2,922 & 599 & 0.000 \\
\hline PDA & 175 & 38.3 & 3.2 & 0.000 & $2,701 \quad 764$ & 0.000 & 74 & 37.9 & 3.4 & 0.000 & 2,724 & 789 & 0.000 & 101 & 38.63 & 3.0 & 0.004 & 2,685 & 749 & 0.000 \\
\hline $\mathrm{CT}$ & 43 & 38.5 & 3.3 & 0.012 & 6738 & 0.000 & 30 & 38.9 & 3.2 & 0.299 & 2,962 & 772 & 0.000 & 13 & 37.53 & 3.5 & 0.003 & 2,678 & 634 & \\
\hline TGA & 302 & 39.5 & 1.9 & 0.115 & $3,160 \quad 584$ & 0.000 & 166 & 39.6 & 2.0 & 0.303 & 3,264 & 588 & 0.097 & 136 & 39.51 & 1.8 & 0.160 & 3,033 & 556 & 00 \\
\hline DORV & 24 & 39.4 & 2.0 & 0.913 & $3,171 \quad 563$ & 0.301 & 12 & 39.3 & 1.6 & 0.825 & 3,409 & 426 & 0.587 & 12 & 39.52 & 2.4 & 0.665 & 2,933 & 597 & 0.073 \\
\hline CTD & 585 & 39.4 & 2.0 & 0.699 & $3,083 \quad 583$ & 0.000 & 331 & 39.4 & 2.1 & 0.802 & 3,169 & 605 & 0.000 & 254 & 39.32 & 2.0 & 0.609 & 2,973 & 536 & 0.000 \\
\hline $\mathrm{A} / \mathrm{SPV}$ & 72 & 39.3 & 2.4 & 0.987 & 3,094706 & 0.007 & 37 & 39.6 & 2.2 & 0.398 & 3,334 & 578 & 0.596 & 35 & 38.92 & 2.60 & 0.477 & 2,841 & 746 & 0.000 \\
\hline A/STV & 13 & 37.3 & 3.7 & 0.000 & 2,616899 & 0.000 & 5 & 36.0 & 5.2 & 0.000 & 2,450 & 1,416 & 0.000 & 8 & 38.12 & 2.50 & 0.166 & 2,720 & 455 & 0.011 \\
\hline EA & 7 & 38.3 & 3.1 & 0.139 & 2,700609 & 0.002 & 6 & 38.2 & 3.4 & 0.111 & 2,725 & 663 & 0.004 & 1 & 39.00 & 0.0 & 0.801 & 2,550 & 0 & 0.154 \\
\hline CAPA & 107 & 38.9 & 2.7 & 0.039 & 3,009716 & 0.000 & 60 & 38.9 & 2.4 & 0.075 & 3,039 & 660 & 0.000 & 47 & 38.93 & 3.10 & 0.308 & 2,972 & 787 & 0.003 \\
\hline RSOD & 199 & 38.9 & 2.7 & 0.006 & $3,003 \quad 727$ & 0.000 & 108 & 39.0 & 2.6 & 0.038 & 3,095 & 705 & 0.000 & 91 & 38.82 & 2.9 & 0.107 & 2,895 & 743 & 0.000 \\
\hline VAS & 56 & 39.1 & 2.4 & 0.545 & $3,038 \quad 529$ & 0.001 & 37 & 39.0 & 2.1 & 0.284 & 3,051 & 516 & 0.002 & 19 & 39.43 & 3.0 & 0.635 & 3,013 & 566 & 0.184 \\
\hline HLH & 73 & 38.7 & 2.9 & 0.004 & $2,960 \quad 644$ & 0.000 & 47 & 38.7 & 3.0 & 0.018 & 3,000 & 659 & 0.000 & 26 & 38.62 & & 0.108 & 2,888 & 622 & 0.002 \\
\hline LSOD & 295 & 38.8 & 2.6 & 0.000 & $3,028 \quad 621$ & 0.000 & 192 & 38.7 & 2.6 & 0.000 & 3,056 & 626 & 0.000 & 103 & 38.92 & 2.80 & 0.118 & 2,977 & 611 & 0.000 \\
\hline OCHD & 181 & 38.2 & 3.0 & 0.000 & $2,828 \quad 731$ & 0.000 & 83 & 38.4 & 2.9 & 0.000 & 2,963 & 703 & 0.000 & 98 & 38.03 & 3.0 & 0.000 & 2,714 & 739 & 0.000 \\
\hline CCHD & 81 & 39.1 & 2.4 & 0.305 & $3,053 \quad 603$ & 0.000 & 42 & 38.5 & 2.6 & 0.003 & 3,045 & 662 & 0.000 & 39 & 39.62 & 2.0 & 0.095 & 3,062 & 540 & 0.114 \\
\hline Total & 3,750 & 38.9 & 2.5 & 0.000 & $3,007 \quad 643$ & 0.000 & 1,901 & 138.9 & 2.5 & 0.000 & 3.092 & 646 & 0.000 & 1,849 & 38.62 & $2.6 \quad$ & 0.000 & 2,920 & 629 & 0.000 \\
\hline
\end{tabular}

Table 4: Number of Singletons, and Rates of Their Preterm Birth (PTB), Low Birthweight (LBW) and Small for Gestational Age (SGA) in Different Study Groups and Population Controls (PC) As Reference

\begin{tabular}{|c|c|c|c|c|c|c|c|c|}
\hline \multirow[t]{2}{*}{ Study groups } & \multirow{2}{*}{$\begin{array}{c}\text { Total } \\
\mathrm{N}\end{array}$} & \multicolumn{2}{|c|}{ Preterm birth (PTB) } & \multicolumn{2}{|c|}{ Low birthweight (LBW) } & \multicolumn{3}{|c|}{ Small for gestational age (SGA) } \\
\hline & & No. $\%$ & OR, $\quad 95 \% \mathrm{CI}$ & No. & $\mathrm{OR}, \quad 95 \% \mathrm{CI}$ & No. & $\%$ & OR, $\quad 95 \% \mathrm{CI}$ \\
\hline $\mathrm{PC}$ & 37,754 & $3,385 \quad 9.0$ & Reference & $2,023 \quad 5.4$ & Reference & 816 & 2.2 & Reference \\
\hline VSD & 1,631 & $192 \mathbf{1 1 . 8}$ & $1.24, \quad 1.06-1.44$ & $257 \quad 15.8$ & $2.98,2.58-3.43$ & 146 & 9.0 & $4.06, \quad 3.36-4.87$ \\
\hline SV & 72 & 11.1 & $1.13, \quad 0.50-2.24$ & 16.7 & $3.10, \quad 1.57-5.63$ & 5 & 6.9 & $3.01, \quad 1.05-6.81$ \\
\hline ASD-II & 454 & $100 \quad \mathbf{2 2 . 0}$ & $2.60, \quad 2.05-3.25$ & $120 \quad 26.4$ & $5.50, \quad 4.40-6.84$ & 37 & 8.1 & $3.49, \quad 2.42-4.88$ \\
\hline CAVC & 75 & $15 \quad 19.5$ & $2.20, \quad 1.04-3.79$ & 20.8 & $4.00, \quad 2.20-6.87$ & 9 & 12.0 & $5.44,2.51-10.44$ \\
\hline PDA & 175 & $42 \quad \mathbf{2 4 . 0}$ & $2.85, \quad 1.98-4.02$ & 37.7 & $9.55, \quad 6.92-13.08$ & 34 & 19.4 & $9.73, \quad 6.51-14.15$ \\
\hline CT & 43 & 18.6 & $2.02 \quad 0.86-4.18$ & 34.9 & $8.62,4.41-16.18$ & 10 & 23.3 & $12.92,5.97-25.63$ \\
\hline TGA & 302 & $23 \quad 7.6$ & $0.79, \quad 0.50-1.19$ & 10.9 & $2.02, \quad 1.37-2.87$ & 26 & 8.6 & 4.04, 2.62-5.97 \\
\hline $\mathrm{TF}$ & 216 & 6.5 & $0.66, \quad 0.36-1.09$ & 13.9 & $2.68, \quad 1.77-3.91$ & 24 & 11.1 & $5.33,3.37-8.06$ \\
\hline DORV & 24 & 8.3 & $0.86, \quad 0.14-2.96$ & 16.7 & $3.26, \quad 0.93-8.79$ & 4 & 16.7 & $8.76, \quad 2.52-23.55$ \\
\hline CTD & 585 & $47 \quad 8.0$ & $0.83, \quad 0.60-1.11$ & 14.0 & $2.69, \quad 2.10-3.40$ & 64 & 10.9 & $5.27,3.98-6.86$ \\
\hline $\mathrm{A} / \mathrm{SPV}$ & 72 & 15.3 & $1.60, \quad 0.79-2.94$ & 18.1 & $3.21, \quad 1.66-5.74$ & 8 & 11.1 & $4.72, \quad 2.07-9.39$ \\
\hline A/STV & 13 & 15.4 & $1.80, \quad 0.28-6.84$ & 38.5 & $11.77,3.43-36.5$ & 4 & 30.8 & $20.71,5.47-65.50$ \\
\hline EA & 7 & 14.3 & $1.72, \quad 0.09-10.39$ & 28.6 & $7.84, \quad 1.09-37.77$ & 1 & 14.3 & $8.05, \quad 0.42-48.22$ \\
\hline CAPA & 107 & 16.8 & $1.93, \quad 1.12-3.15$ & 24.3 & $4.86,3.03-7.56$ & 13 & 12.1 & $5.17,2.72-9.03$ \\
\hline RSOD & 199 & 16.1 & $1.80, \quad 1.20-2.60$ & 23.1 & $4.61,3.24-6.43$ & 26 & 13.1 & $5.79, \quad 3.70-8.69$ \\
\hline VAS & 56 & 8.9 & $0.88, \quad 0.30-2.02$ & 12.5 & $2.17, \quad 0.89-4.56$ & 3 & 5.4 & $2.22, \quad 0.54-6.10$ \\
\hline HLH & 73 & $\mathbf{1 7 . 8}$ & $2.13, \quad 1.11-3.78$ & 15.1 & $3.02, \quad 1.49-5.57$ & 2 & 2.7 & $1.21, \quad 0.20-3.89$ \\
\hline OCAA & 54 & 14.8 & $1.49, \quad 0.65-3.01$ & 22.2 & $4.04, \quad 2.01-7.55$ & 4 & 7.4 & $2.91, \quad 0.87-7.20$ \\
\hline LSOD & 295 & 13.2 & $1.42, \quad 1.00-1.98$ & 14.9 & $2.77, \quad 1.97-3.80$ & 14 & 4.7 & $1.99, \quad 1.10-3.31$ \\
\hline OCHD & 181 & $44 \quad \mathbf{2 4 . 3}$ & $3.02, \quad 2.12-4.25$ & 28.7 & $6.25,4.43-8.68$ & 19 & 10.5 & $4.51,2.68-7.17$ \\
\hline $\mathrm{CCHD}$ & 81 & $9 \quad 11.1$ & $1.25, \quad 0.58-2.37$ & 13.6 & $2.66, \quad 1.32-4.86$ & 6 & 7.4 & $3.40, \quad 1.31-7.26$ \\
\hline Total & 3,750 & $528 \quad 14.1$ & $1.51,1.36-1.67$ & $\begin{array}{|ll|}706 & \mathbf{1 8 . 8} \\
\end{array}$ & $3.68, \quad 3.34-4.04$ & 360 & 9.6 & $4.39, \quad 3.85-5.00$ \\
\hline
\end{tabular}

Bold numbers show significant associations 
Table 5: Number of Male Singletons, and Rates of Their Preterm Birth (PTB), Low Birthweight (LBW) and Small for Gestational Age (SGA) in Different Study Groups and Population Controls (PC) As Reference

\begin{tabular}{|c|c|c|c|c|c|c|c|c|c|c|}
\hline \multirow[t]{2}{*}{ Study groups } & \multirow{2}{*}{$\begin{array}{c}\text { Total } \\
\mathrm{N}\end{array}$} & \multicolumn{3}{|c|}{ Preterm birth (PTB) } & \multicolumn{3}{|c|}{ Low birthweight (LBW) } & \multicolumn{3}{|c|}{ Small for gestational age (SGA) } \\
\hline & & No. & $\%$ & OR, $95 \%$ CI & No. & $\%$ & $\begin{array}{ll}\mathrm{OR}, & 95 \% \mathrm{CI}\end{array}$ & No. & $\%$ & OR, $\quad 95 \%$ CI \\
\hline $\mathrm{PC}$ & 25,540 & 1,99 & 68.1 & Reference & 1,15 & 4.7 & Reference & 432 & 1.8 & Reference \\
\hline VSD & 784 & 83 & 10.6 & $1.25, \quad 0.98-1.57$ & 117 & 14.9 & 3.29, $2.66-4.03$ & 73 & 9.3 & $5.39,4.12-6.95$ \\
\hline SV & 38 & 5 & 13.2 & $1.52, \quad 0.52-3.60$ & 7 & 18.4 & $4.05,1.61-8.87$ & 3 & 7.9 & $4.38, \quad 1.05-12.35$ \\
\hline ASD-II & 218 & 39 & 17.9 & $2.23, \quad 1.54-3.14$ & 45 & 20.6 & $4.50, \quad 3.16-6.29$ & 15 & 6.9 & $3.61,2.02-6.00$ \\
\hline CAVC & 31 & 4 & 12.9 & $1.53, \quad 0.45-3.95$ & 6 & 19.4 & 4.30, $1.57-9.99$ & 5 & 16.1 & 9.84, 3.29-23.98 \\
\hline PDA & 74 & 20 & 27.0 & $3.85, \quad 2.23-6.39$ & 27 & 36.5 & $10.86,6.59-17.57$ & 11 & 14.9 & $9.18,4.53-16.95$ \\
\hline $\mathrm{CT}$ & 30 & 4 & 13.3 & $1.41, \quad 0.41-3.67$ & 11 & 36.7 & $10.19,4.58-21.60$ & 8 & 26.7 & $18.80,7.74-41.29$ \\
\hline TGA & 166 & 16 & 9.6 & $1.15, \quad 0.65-1.87$ & 14 & 8.4 & $1.74, \quad 0.95-2.92$ & 9 & 5.4 & $3.09, \quad 1.45-5.76$ \\
\hline $\mathrm{TF}$ & 123 & 9 & 7.3 & $0.81, \quad 0.38-1.52$ & 18 & 14.6 & $3.22, \quad 1.87-5.24$ & 14 & 11.4 & $6.61,3.58-11.31$ \\
\hline DORV & 12 & 1 & 8.3 & $0.84, \quad 0.05-4.93$ & 0 & 00.0 & $0.00, \quad 0.00-1.21$ & 0 & 0.0 & $0.00, \quad 0.00-1638.50$ \\
\hline CTD & 331 & 30 & 9.1 & $1.04, \quad 0.69-1.49$ & 43 & 13.0 & $2.78, \quad 1.97-3.82$ & 31 & 9.4 & $5.43,3.63-7.86$ \\
\hline $\mathrm{A} / \mathrm{SPV}$ & 37 & 5 & 13.5 & $1.56, \quad 0.53-3.71$ & 3 & 8.1 & $1.50, \quad 0.36-4.24$ & 1 & 2.7 & $1.29, \quad 0.07-6.05$ \\
\hline A/STV & 5 & 1 & 20.0 & $3.56, \quad 0.18-24.27$ & 3 & 60.0 & $44.91,7.34-344.8$ & 2 & 40.0 & $47.13,6.14-288.44$ \\
\hline EA & 6 & 1 & 16.7 & $2.18, \quad 0.11-14.02$ & 2 & 33.3 & $11.52,1.54-61.26$ & 1 & 16.7 & $12.86,0.66-81.52$ \\
\hline CAPA & 60 & 9 & 15.0 & $1.91, \quad 0.87-3.72$ & 13 & 21.7 & $4.87, \quad 2.49-8.89$ & 7 & 11.7 & $5.80, \quad 2.35-12.24$ \\
\hline RSOD & 108 & 16 & 14.8 & 1.86, $1.04-3.09$ & 21 & 19.4 & $4.39, \quad 2.62-7.02$ & 11 & 10.2 & $5.30, \quad 2.63-9.64$ \\
\hline VAS & 37 & 3 & 8.1 & $0.87, \quad 0.21-2.45$ & 4 & 10.8 & $2.11, \quad 0.62-5.40$ & 2 & 5.4 & $2.80, \quad 0.45-9.35$ \\
\hline HLH & 47 & 7 & 14.9 & $\begin{array}{ll}1.93, & 0.78-4.08\end{array}$ & 6 & 12.8 & $2.91,1.10-6.42$ & 1 & 2.1 & $1.16, \quad 0.07-5.36$ \\
\hline $\mathrm{COA}$ & 71 & 9 & 12.7 & $\begin{array}{ll}1.60, & 0.74-3.08\end{array}$ & 9 & 12.7 & $2.70, \quad 1.24-5.22$ & 2 & 2.8 & $1.39, \quad 0.23-4.47$ \\
\hline OCAA & 37 & 5 & 13.5 & $1.54, \quad 0.52-3.65$ & 8 & 21.6 & $4.82, \quad 2.03-10.21$ & 2 & 5.4 & $4.26, \quad 1.02-11.99$ \\
\hline LSOD & 192 & 24 & 12.5 & $1.51, \quad 0.95-2.28$ & 27 & 14.1 & $3.02, \quad 1.95-4.51$ & 8 & 4.2 & $2.16, \quad 0.97-4.16$ \\
\hline OCHD & 83 & 19 & 22.9 & $3.1, \quad 1.79-5.12$ & 18 & 21.7 & $4.88, \quad 2.76-8.20$ & 5 & 6.0 & $3.04, \quad 1.06-6.89$ \\
\hline $\mathrm{CCHD}$ & 42 & 7 & 16.7 & $2.3, \quad 0.93-4.91$ & 7 & 16.7 & $3.90, \quad 1.57-8.39$ & 4 & 9.5 & $5.36,1.59-13.56$ \\
\hline Total & 1,901 & 247 & $\mathbf{1 3 . 0}$ & $1.55, \quad 1.34-1.79$ & 318 & 16.7 & $3.69, \quad 3.22-4.23$ & 166 & 8.7 & $4.93,4.07-5.94$ \\
\hline
\end{tabular}

Bold numbers show significant associations

Table 6: Number of Female Singletons, and Rates of Their Preterm Birth (PTB), Low Birthweight (LBW) and Small for Gestational Age (SGA) in Different Study Groups and Population Controls (PC) As Reference

\begin{tabular}{|c|c|c|c|c|c|c|c|c|c|c|c|}
\hline \multirow[t]{2}{*}{ Study groups } & \multirow[t]{2}{*}{ Total N } & \multicolumn{3}{|c|}{ Preterm birth (PTB) } & \multicolumn{3}{|c|}{ Low birthweight (LBW) } & \multicolumn{4}{|c|}{ Small for gestational age (SGA) } \\
\hline & & No. & $\%$. & $\mathrm{OR}, 95 \% \mathrm{CI}$ & No. & $\%$ & OR, $95 \% \mathrm{CI}$ & No. & $\%$ & OR, & $95 \% \mathrm{CI}$ \\
\hline PC & 13,214 & 1,38 & 10.5 & Reference & 864 & 6.5 & Reference & 384 & 2.9 & Refer & \\
\hline VSD & 847 & 109 & 12.9 & $1.13,0.91-1.38$ & 140 & 16.5 & $2.46,2.01-2.99$ & 73 & 8.6 & 2.80 , & $2.14-3.63$ \\
\hline SV & 34 & 3 & 8.8 & $0.74,0.18-2.10$ & 5 & 14.7 & $2.11,0.71-5.11$ & 2 & 5.9 & 1.81 , & $0.29-6.06$ \\
\hline ASD-II & 236 & 61 & 25.8 & 2.37, 1.96-3.59 & 75 & 31.8 & $5.71,4.24-7.63$ & 22 & 9.3 & 2.92 & $1.80-4.52$ \\
\hline CAVC & 46 & 11 & 23.9 & $2.40,1.15-4.63$ & 10 & 21.7 & $3.42,1.58-6.80$ & 4 & 8.7 & 2.86, & $0.85-7.21$ \\
\hline PDA & 101 & 22 & 21.8 & $2.06,1.24-3.28$ & 39 & 38.6 & $7.60,4.95-11.52$ & 23 & 22.8 & 8.29, & 4.99-13.27 \\
\hline CT & 13 & 4 & 30.8 & $3.77,1.01-11.70$ & 4 & 30.8 & 7.34, $1.94-23.32$ & 2 & 15.4 & 7.04, & $1.06-27.55$ \\
\hline $\mathrm{TF}$ & 93 & 5 & 5.4 & $0.47,0.17-1.05$ & 12 & 12.9 & $2.09,1.07-3.73$ & 10 & 10.8 & 4.01, & 1.92-7.49 \\
\hline DORV & 12 & 1 & 8.3 & $0.73,0.04-3.81$ & 4 & 33.3 & 7.66, $1.97-25.58$ & 4 & 33.3 & 18.67 & 4.78-63.42 \\
\hline CTD & 254 & 17 & 6.7 & $0.58,0.34-0.92$ & 39 & 15.4 & $2.50,1.73-3.52$ & 33 & 13.0 & 4.88, & 3.27-7.08 \\
\hline A/SPV & 35 & 6 & 17.1 & $1.51,0.56-3.45$ & 10 & 28.6 & 4.69, 2.09-9.74 & 7 & 20.0 & 7.15, & 2.82-15.84 \\
\hline A/STV & 8 & 1 & 12.5 & $1.02,0.05-5.94$ & 2 & 25.0 & $3.81,0.53-17.63$ & 2 & 25.0 & 9.52 & $1.34-43.88$ \\
\hline EA & 1 & 0 & 0.0 & $0.00,0.00-\mathrm{inf}$ & 0 & 0.0 & $0.00,0.00$-inf & 0 & 0.0 & 0.00 & 0.00 -Inf \\
\hline CAPA & 47 & 9 & 19.1 & $1.88,0.85-3.76$ & 13 & 27.7 & $4.76,2.37-9.03$ & 6 & 12.8 & 4.41, & $1.65-9.88$ \\
\hline RSOD & 91 & 16 & 17.6 & $1.64,0.91-2.76$ & 25 & 27.5 & $4.60,2.80-7.35$ & 15 & 16.5 & 5.84, & $3.17-10.09$ \\
\hline VAS & 19 & 2 & 10.5 & $0.89,0.14-3.14$ & 3 & 15.8 & $2.38,0.54-7.47$ & 1 & 5.3 & 1.68, & $0.09-8.44$ \\
\hline HLH & 26 & 6 & 23.1 & $2.45,0.89-5.84$ & 5 & 19.2 & $3.12,1.02-7.89$ & 1 & 3.8 & 1.22, & $0.07-5.87$ \\
\hline OCAA & 17 & 3 & 17.6 & $1.44,0.33-4.50$ & 4 & 23.5 & $3.07,0.84-9.03$ & 1 & 5.9 & 1.47, & $0.08-7.43$ \\
\hline LSOD & 103 & 15 & 14.6 & $1.30,0.72-2.19$ & 17 & 16.5 & $2.45,1.39-4.08$ & 6 & 5.8 & 1.81, & $0.70-3.86$ \\
\hline OCHD & 98 & 25 & 25.5 & $2.74,1.69-4.31$ & 34 & 34.7 & $6.62,4.23-10.19$ & 14 & 14.3 & 4.61, & 2.45-8.06 \\
\hline CCHD & 39 & 2 & 5.1 & $0.43,0.07-1.43$ & 4 & 10.3 & $1.54,0.46-3.92$ & 2 & 5.1 & 1.71, & $0.28-5.67$ \\
\hline Total & 1,849 & 281 & 15.2 & $1.37,1.19-1.58$ & 388 & 21.0 & $3.34,2.92-3.83$ & 194 & 10.5 & 3.53, & $2.94-4.24$ \\
\hline
\end{tabular}

Bold numbers show significant associations

\section{Discussion}

The major findings of the study suggested that in general cases with different CHD-entities had a shorter gestational age at delivery and lower mean birth weight, and these variables associated with a higher rate of PTB and particularly with a much higher rate of LBW and SGA.

Three CHD-entities and/or groups are worth more discussion.

i) Cases with ASD-II had localized small defect of an atrial septum (Fyler 1992, Vereczkey et al. 2013), nevertheless, their mean gestational age was $1.2 \mathrm{wk}$ shorter and their mean birth weight was $412 \mathrm{~g}$ smaller than population controls. The rate of PTB, LBW and SGA was 2.4. 4.9 and 3.7 fold higher in cases with ASD-II than in population controls, respectively. Thus intrauterine growth restriction was characteristic for ASD-II, mainly in female cases. There was no robust difference in the mean gestational age of males and females (1.1 vs. $1.2 \mathrm{wk}$ ), but the mean birth weight ( $431 \mathrm{~g}$ ) was smaller in females than in males $(314 \mathrm{~g})$. The rate of PTB ( 2.5 vs. 2.2 fold) and LBW (4.9 vs. 4.4 fold) was higher in female cases that in male cases compared to their appropriate sex controls. However, this difference was reverse in the rate of SGA (3.2 vs. 3.8 fold).

ii) TGA is severe CHD-entity, nevertheless, newborns with TGA had somewhat longer gestational age $(0.1 \mathrm{wk})$, but mean birth weight was 66 and $160 \mathrm{~g}$ smaller in male and female cases than in male and female controls, respectively. There was no higher rate of PTB in male and female cases, LBW rate was higher only in female cases. However, SGA showed a higher rate in both sexes. Thus TGA had no effect for the gestational age and PTB, but TGA was associated with intrauterine growth restriction, mainly in female cases.

iii) In general, the four types of LSOD had no higher rate of PTB in male and female cases, and only HLH had a higher rate of LBW in both sexes. In addition, the rate of LBW was higher in males with COA and OCAA, while higher rate of SGA occurred only in male cases with OCAA. Thus intrauterine growth restriction occurred mainly in males.

The birth prevalence of CHDs was $7.06+0.91$ per 1000 in Budapest, 1963-1965 based on the HCAR completed with active search of records in all pediatric and pathologic institutions (Czeizel et al. 1972). However, the birth prevalence of CHDs was $10.2+2.1$ per 1000 in a Hungarian population-based study of a 
country region, when each individual child was examined personally by a pediatric cardiologist in 1971-1972 or the autopsy report was evaluated in dead children (Mészáros et al. 1975). Thus the Hungarian rate of CHDs corresponded to the internationally reported figures (Hoffman et al. 2004, Reller et al. 2008, Dolk et al. 2011). In general, the sex of cases with different CHD-entities was also similar in our material than in other studies (Ferencz et al 1993, Reller et al. 2008). However, our study showed the importance of sex in the birth outcomes of some CHD-entities. The birth outcomes of cases with different CHD-entities/groups were presented in The Baltimore-Washington Infant Study 19811989 (Ferencz et al. 1997). For example, mean gestational age and mean birth weight was $38.1 \pm 0.3 \mathrm{wk}$ and $2,939 \pm 60 \mathrm{~g}$ in cases with ASD-II while these figures were $38.2 \pm 3.1$ wk and $2.870 \pm$ $737 \mathrm{~g}$ in our material, but the mean gestational age and birth weight of controls was $39.6 \pm 0.1 \mathrm{wk}$ and $3,351 \pm 10$ in The Baltimore-Washington Infant Study. The OR (95\% CI) for SGA was 2.7 (1.7-4.1) in The Baltimore-Washington Infant Study [13] and 3.5 (2.4-4.9) in our material, respectively.

TGA was also not associated with a higher rate of PTB in The Baltimore-Washington Infant Study (Ferencz et al. 1997) as in our study, and the birth outcomes of cases with LSOG group were also similar in the two study material.

Only mean gestational age and birth weight were published in Metropolitan Atlanta Congenital Defects Program (Reller et al 2008). The mean gestational age was $38.0 \mathrm{wk}$ in cases with PDA similar to $38.3 \mathrm{wk}$ in our material. However, there was some difference in the mean gestational age ( 38.5 vs. $39.5 \mathrm{wk})$ of cases with TGA in there and our material though the difference in mean birth weight was small (3,173 vs. 3,160 g).

Some difference in the birth outcomes of cases with different CHD-entities may be connected with the different ascertainment and selection of cases in different studies. We evaluated the severe cases with lethal outcome or after catheter diagnoses or surgical correction, thus or cases represented the tip of an iceberg. Thus the difference in birth outcomes of cases with different severe CHDentities suggests the different pathogenesis of CHD-entities.

The question is why several CHD-entities are manifesting more frequently in newborns with PTB and mainly with LBW/SGA, because intrauterine growth restriction was observed in most CHD-entities. Three options are worth discussing.

i) It is difficult to believe that a localized CA in the heart such as ASD-II can induce intrauterine fetal growth restriction because the fetal heart has a passive role before birth without pulmonary circulation. Nevertheless, our data indicate the effect of a fetal heart defects for fetal development. First Rosenthal (Rosenthal 1996) suggested that intrauterine life hemodynamic alterations due to the CHD may affect size and growth pattern of fetuses.

ii) Possible confounding factors such as maternal age, birth order and socioeconomic status have to evaluate as well. Maternal age and birth order were considered for the calculation of adjusted $\mathrm{p}$ and $\mathrm{OR}$ values in this study. The poor socioeconomic status is associated with lower birth weight in general and e.g. in cases with VSD (Vereczkey et al. 2012). However, this effect cannot explain the differences among CHD-entities/groups. Drinking and mainly smoking habits are also important. However, our studies showed a similar rate of these lifestyle factors in the mothers of cases with CHD, thus these adverse lifestyle factors cannot explain totally the difference in fetal growth restriction of fetuses (Czeizel et al. 2003). Maternal diseases and related drug treatments were also analyzed without any association with higher risk of intrauterine growth restriction of cases with CHD (Ács et al. 2010).

iii) CHDs and intrauterine growth restriction as two developmental errors may have a common route. Recently, the association of some gene polymorphisms with fetal growth and birth weight was shown (Freathy et al. 2010), thus it would be interesting to test the effect of these gene variants in the origin of CHDs.

The strengths of our study are connected with the large population-based data set of the HCCSCA, including 3,750 singleton live-born cases with CHD and 37,741 singleton liveborn population controls without CAs in the ethnically homogeneous Hungarian (Caucasian) population. Cases with CHD were reported by medical doctors and reported diagnoses were critically checked in the HCAR (Czeizel 1997, Czeizel et al. 2014). In addition, the validity of CHD-diagnoses has been improved due to the available medical records on $3.5+2.1$ months after the birth in the data set of the HCCSCA (Czeizel et al. 2001) and finally due to the follow-up of our cases in cardiologic institutes. We did our best to work with cases with CHD-entities as homogeneous as possible; therefore, cases with syndromic/multiple CA including CHD were excluded and finally only cases after catheter diagnosis or surgical correction or lethal outcome based on medical documents were planned to evaluate in the study. The data of birth outcomes were medically recorded.

However, there were some weaknesses of our study. (i) The diagnosis of CHDs was accomplished during the study period (1980-1996), i.e. mainly before the spectacular improvement of medical technology, particularly echocardiography. However, our cases with CHD were selected until the end of third postnatal month and mainly surgically corrected or lethal cases were included in the study, thus mainly more severe CHDs cases were evaluated. (ii) Another weakness of our study is the lack of data regarding smoking and drinking habit in the total material of mothers of cases and controls. Our previous validation study showed the low reliability of retrospective self-reported information on these habits in control mothers and mainly in case mothers (Czeizel et al. 2003). Thus only the family consensus data after the home visit in a subsample of cases and controls were evaluated; therefore, we cannot consider the smoking and drinking habit as confounder in the mothers of all cases and controls.

In conclusion, our findings showed significant difference in the birth outcomes of cases with CHD compared to their population controls. The higher rate of LBW and SGA indicates intrauterine fetal growth restriction in several CHD-entities and the sex of cases has some effect for their fetal development. The intrauterine growth restriction as a general developmental process may have some role in the origin of some CHD-entities. Nevertheless, birth outcomes of different CHD-entities were different and these findings confirmed the different pathogenesis of CHD-entities.

\section{Acknowledgement}

This project was supported by the Hungarian Egészségügyi Tudományos Tanács Pályázati Irodája (Grant Office of Scientific Committee of Health Ministry) and Versys Clinics, Human Reproduction Institute, Budapest, Hungary.

\section{Declaration of interest}

The authors report no conflicts of interest. The authors alone are responsible for the content and writing of the paper.

\section{References}

[1] Hoffman JI, Kaplan S \& Liberthson RR (2004) Prevalence of congenital heart disease. American Heart Journal 147, 425-439. http://dx.doi.org/10.1016/i.ahj.2003.05.003.

[2] Dolk H, Loane M \& Garne E; European Surveillance of ongenital Anomalies (EUROCAT) Working Group (2011) Congenital heart defects in Europe: prevalence and perinatal mortality, 2000 to 2005. Circulation 123 , 841-849. http://dx.doi.org/10.1161/CIRCULATIONAHA.110.958405.

[3] Reller MD, Strickland MJ, Riehle-Colarusso T, Mahle WT \& Correa A (2008) Prevalence of congenital heart defects in metropolitan Atlan- 
ta, 1998-2005. The Journal of Pediatrics 153, 807-813. http://dx.doi.org/10.1016/j.jpeds.2008.05.059.

[4] Czeizel A, Kamarás J, Balogh O \& Szentpéteri J (1972) Incidence of congenital heart defects in Budapest. Acta Paediatrica Academiae Scientiarum Hungaricae 13, 191-202.

[5] Mészáros M, Nagy A \& Czeizel A (1975) Incidence of congenital heart disease in Hungary. Human Heredity 25, 513-519. http://dx.doi.org/10.1159/000152769.

[6] Fulton DR (2008) congenital heart diseases in children and adolescent In Hurst's the Heart (Fuster V, Walsh A, O'Rourke RA, Poole-Wilson P eds.), McGraw Hill Medical, New York, pp. 1855-1921.

[7] Cleves MA, Ghaffar S, Zhao W, Mosley BS \& Hobbs CA (2003) First-year survival of infants born with congenital heart defects in $\mathrm{Ar}$ kansas (1993-1998): a survival analysis using registry data. Birth Defects Research. Part A, Clinical and Molecular Teratology 67, 662-668. http://dx.doi.org/10.1002/bdra.10119.

[8] Pierpont ME, Basson CT, Benson DW Jr, Gelb BD, Giglia TM, Goldmuntz E, McGee G, Sable CA, Srivastava D \& Webb CL; American Heart Association Congenital Cardiac Defects Committee, Council on Cardiovascular Disease in the Young (2007) Genetic basis for congenital heart defects: current knowledge: a scientific statement from the American Heart Association Congenital Cardiac Defects Committee, Council on Cardiovascular Disease in the Young: endorsed by the American Academy of Pediatrics. Circulation 115, 3015-3038. http://dx.doi.org/10.1161/CIRCULATIONAHA.106.183056.

[9] Jenkins KJ, Correa A, Feinstein JA, Botto L, Britt AE, Daniels SR, Elixson M, Warnes CA \& Webb CL; American Heart Association Council on Cardiovascular Disease in the Young (2007) Noninherited risk factors and congenital cardiovascular defects: current knowledge: a scientific statement from the American Heart Association Council on Cardiovascular Disease in the Young: endorsed by the American Academy of Pediatrics. Circulation 115, 2995-3014. http://dx.doi.org/10.1161/CIRCULATIONAHA.106.183216.

[10]Czeizel AE, Rockenbauer M, Siffel Cs \& Varga E (2001) Description and mission evaluation of the Hungarian Case-Control Surveillance of Congenital Abnormalities, 1980-1996. Teratology 63, 176-185. http://dx.doi.org/10.1002/tera.1032.

[11] Botto LD, Lin AE, Riehle-Colarusso T, and Malik S \& Correa a (2007) Seeking cause: Classifying and evaluating congenital heart defects in etiologic studies. Birth Defects Research. Part A, Clinical and $\begin{array}{llll}\text { Molecular } & \text { Teratology } & 79, & 714-727\end{array}$ http://dx.doi.org/10.1002/bdra.20403.

[12]Ferencz C, Loffredo CA, Rubin JD \& Magee CA (1993) Epidemiology of Congenital Heart Diseases. The Baltimore-Washington Infant Study: 1981-1989. Future Publ Co., Mount Kisco, New York.

[13]Ferencz C, Loffredo CA, Correa-Villasenor A \& Wilson PD (1997) Genetic and Environmental Risk Factors of Major Cardiovascular Malformations: The Baltimore-Washington Infant Study: 1981-1989. Future Publ Co., Armonk, New York.

[14]Czeizel AE (1997) the first 25 years of the Hungarian congenital abnormality registry. Teratology 55, 299-305. http://dx.doi.org/10.1002/(SICI)1096-9926(199705)55:5<299::AIDTERA1>3.0.CO;2-V.

[15] Czeizel AE, Métneki J \& Béres J (2014) 50 years of the Hungarian Congenital Abnormality Registry. Congenital Anomalies (Kyoto) 54 22-29. http://dx.doi.org/10.1111/cga.12025.

[16]Czeizel AE, Intődy Z \& Modell B (1993) what proportion of congenital abnormalities can be prevented? British Medical Journal 306, 499 503. http://dx.doi.org/10.1136/bmj.306.6876.499.

[17]Czeizel AE, Petik D \& Vargha P (2003) Validation studies of drug exposures in pregnant women. Pharmacoepidemiology and Drug Safety 12, 409-416. http://dx.doi.org/10.1002/pds.766.

[18]Czeizel AE \& Vargha P (2004) Periconceptional folic ac$\mathrm{id} /$ multivitamin supplementation and twin pregnancy. American Journal of Obstetrics and Gynecology 191, 790-794. http://dx.doi.org/10.1016/j.ajog.2004.02.018.

[19]Ács N, Bánhidy F, Puho E \& Czeizel AE (2005) maternal influenza during pregnancy and risk of congenital abnormalities in offspring. Birth Defects Research. Part A, Clinical and Molecular Teratology 73 , 989-996. http://dx.doi.org/10.1002/bdra.20195.

[20]Fyler DC (1992) Atrial septal defect secundum. In Nadas' Pediatric Cardiology (Fyler DC ed.), Mosby Year Book, Boston, pp. 513-524.

[21]Vereczkey A, Kosa Z, Csáky-Szunyogh M, Urbán R \& Czeizel AE (2013) Birth outcomes of cases with isolated atrial septal defect type II - a population-based case-control study. Acta Obstetricia ET Gyne$\begin{array}{llll}\text { cologica } & \text { Scandinavica } & \text { 824-829. }\end{array}$ http://dx.doi.org/10.1111/aogs.12062.
[22]Rosenthal GL (1996) Patterns of prenatal growth among infants with cardiovascular malformations: possible fetal hemodynamic effects. American Journal of Epidemiology 143, 505-513. http://dx.doi.org/10.1093/oxfordjournals.aje.a008771.

[23] Vereczkey A, Kosa Zs, Csáky-Szunyogh M, Urbán R \& Czeizel AE (2012) Ventricular septal defect in function of maternal sociodemographic aspects. Central European Journal of Medicine 7, 511-522. http://dx.doi.org/10.2478/s11536-012-0024-x.

[24]Czeizel AE, Petik D \& Puho E (2003) Smoking and alcohol drinking during pregnancy. The reliability of retrospective maternal selfreported information. Central European Journal of Public Health 12, 179-183.

[25] Ács N, Bánhidy F \& Czeizel AE (2010) Congenital Abnormalities and Preterm Birth Related to Maternal Illnesses During Pregnancy. Springer Verlag, Dordrecht, Heidelberg, London, New York. http://dx.doi.org/10.1007/978-90-481-8620-4.

[26] Freathy RM, Mook-Kanamori DO, Sovio U, Prokopenko I, Timpson NJ, Berry DJ, Warrington NM, Widen E, Hottenga JJ, Kaakinen M, Lange LA, Bradfield JP, Kerkhof M, Marsh JA, Mägi R, Chen CM, Lyon HN, Kirin M, Adair LS, Aulchenko YS, Bennett AJ, Borja JB, Bouatia-Naji N, Charoen P, Coin LJ, Cousminer DL, de Geus EJ, Deloukas P, Elliott P, Evans DM, Froguel P; Genetic Investigation of ANthropometric Traits (GIANT) Consortium, Glaser B, Groves CJ, Hartikainen AL, Hassanali N, Hirschhorn JN, Hofman A, Holly JM, Hyppönen E, Kanoni S, Knight BA, Laitinen J, Lindgren CM; MetaAnalyses of Glucose and Insulin-related traits Consortium, McArdle WL, O'Reilly PF, Pennell CE, Postma DS, Pouta A, Ramasamy A, Rayner NW, Ring SM, Rivadeneira F, Shields BM, Strachan DP, Surakka I, Taanila A, Tiesler C, Uitterlinden AG, van Duijn CM; Wellcome Trust Case Control Consortium, Wijga AH, Willemsen G, Zhang H, Zhao J, Wilson JF, Steegers EA, Hattersley AT, Eriksson JG Peltonen L, Mohlke KL, Grant SF, Hakonarson H, Koppelman GH, Dedoussis GV, Heinrich J, Gillman MW, Palmer LJ, Frayling TM, Boomsma DI, Davey Smith G, Power C, Jaddoe VW, Jarvelin MR; Early Growth Genetics (EGG) Consortium \& McCarthy MI (2010) Variants in ADCY5 and near CCNL1 are associated with fetal growth and birth weight. Nature Genetics 42, 430-435. http://dx.doi.org/10.1038/ng.567. 